Conclusions The in vitro gene expression levels of individual chemokines (CXCL1 and CCL2) determines the MDSC infiltration in vivo into the TME. Targeting the chemokine-receptor axis of MDSC subpopulations could be a promising approach in the treatment of pancreatic cancer.

Funding The project was supported by the Deutsche Forschungsgemeinschaft (DFG, German Research Foundation) Projektnummer 329628492 - SFB 1321 and the Förderprogramm für Forschung und Lehre (FöFoLe) funded by the Ludwig-Maximilians-Universität München.

Disclosure Information C. Rambuscheck: None. P. Metzger: None. C. Hörth: None. R. Hennel: None. S. Bärthel: None. C. Falcomatà: None. K. Lauber: None. S. Endres: None. D. Saur: None. M. Schnurr: None. L.M. König: None.

\section{P03.12 IMMUNOPHENOTYPING OF LIVER AND LUNG METASTASES IN COLORECTAL CANCER}

F Schlüter*, K Dötzer, M Prüfer, A Bazhin, J Werner, B Mayer. Department of General, Visceral and Transplant Surgery, LMU, Munich, Germany

\subsection{6/jitc-2020-ITOC7.51}

Background Immunotherapy is an attractive strategy for second-and further-line treatment of metastatic colorectal cancer (mCRC). However, currently immune checkpoint-inhibitors are limited to the small subgroup of dMMR-MSI-H patients. Therefore additional patient stratification markers for immunotherapy independent from the MSI-status are urgently required.

Materials and Methods In this study the immune infiltrate of 53 liver and 15 lung mCRC were immunhistochemically analysed and correlated with clinicopathological parametes related to the primary tumor and the metastatic lesion and the PDL1 status. The CD3, CD8 and PD-1 infiltrate were quantitatively counted positive cells $/ \mathrm{mm}^{2}$ in three different topographic regions, namely invasion margin (IM), stromal (S) and intratumoral (IT). PD-L1 expression was semiquantitatively evaluated with the cut off $>1 \%$. The statistical analyses were performed by the Fisher`s exact-Test (two-tailed).

Results In liver metastases (LM) a high immune infiltrate of CD3 IM, CD3 S, CD8 S and PD-1 S, significantly correlated with an advanced stage ( $\mathrm{pN} 1 / 2 ; \mathrm{cM} 1)$ of the primary tumor. Independent of the type of adjuvant chemotherapy, a significantly higher fraction of CD3 + and CD8 + cells was found at the invasion margin of LM. In contrast, neoadjuvant chemotherapy induced a reduction of PD-L1 expression. Interestingly, a high CD8 IT infiltrate and a high PD-L1 expression correlated with KRAS wildtype. In addition, a high CD8 IT infiltrate and a high PD-L1 expression were found in confined LM, defined as less than two segments and unilobular distribution. A high PD-L1 expression was accompanied by a strong infiltrate of CD3, CD8 and PD-1 positive cells. In contrast, the small cohort of lung metastases showed a significant correlation for a high CD8 S infiltrate and a PD-1 IM infiltrate with right-sided metastases. Additionally, a high PD1 IM infiltrate could be seen after neoadjuvant chemotherapy in lung metastases.

Conclusions Chemotherapeutic treatment strategy might have an impact on subsequent immunotherapy. Combination of anti-EGFR inhibitors with immunotherapy and CD3/PD-L1 Bispecific antibodies are promising options to treat liver and lung metastasis of CRC.
Disclosure Information F. Schlüter: None. K. Dötzer: None. M. Prüfer: None. A. Bazhin: None. J. Werner: None. B. Mayer: None.

\section{P03.13 AGE-INDUCED CHANGES IN ANTI-TUMOR IMMUNITY ALTER THE TUMOR IMMUNE INFILTRATE AND REDUCE RESPONSE TO IMMUNE-ONCOLOGY TREATMENTS}

S Sitnikova*, M Morrow, V Valge-Archer, RW Wilkinson, MJ Robinson, SJ Dovedi. AstrZeneca, Cambridge, UK

\subsection{6/jitc-2020-ITOC7.52}

Background Immuno-Oncology research relies heavily on murine syngeneic tumor models. However, whilst the median age for a cancer diagnosis is 65 years or older, for practical purposes the majority of preclinical studies are conducted in young mice, despite the fact that ageing has been shown to have a significant impact on the immune response.

Materials and Methods Using aged mice bearing CT26 tumors, we analysed how aging impacts the immune composition of the tumor, spleen and tumor-draining lymph nodes by flow cytometry.

Results We found many age-related changes between aged (60-72 weeks old) and young (6-8 weeks old) mice, such as a reduction in the naïve $\mathrm{T}$ cell population and a decreased CD8/Treg ratio in aged animals. Profiling of co-inhibitory and co-stimulatory receptor expression levels on immune cells in aged versus young mice also identified altered expression profiles in both the periphery and tumor. We hypothesised that these differences may contribute to impaired anti-cancer immune responses in aged mice. To investigate this, we compared the anti-tumor efficacy of immune checkpoint blockade (PD-L1 and CTLA-4) and T-cell costimulation (OX-40) in aged versus young mice. Our data demonstrate that aged mice retained their capacity to generate effective anti-tumor immune responses, albeit often attenuated when compared to the responses observed in young mice.

Conclusions These differences highlight the potential importance of age-related immunological changes in assessing and refining the translational insights gained from preclinical mouse models.

Disclosure Information S. Sitnikova: A. Employment (full or part-time); Significant; AstraZeneca. M. Morrow: A. Employment (full or part-time); Significant; AstraZeneca. V. ValgeArcher: A. Employment (full or part-time); Significant; AstraZeneca. R.W. Wilkinson: A. Employment (full or part-time); Significant; AstraZeneca. M.J. Robinson: A. Employment (full or part-time); Significant; AstraZeneca. S.J. Dovedi: A. Employment (full or part-time); Significant; AstraZeneca.

\section{P03.14 PRECLINICAL CASE STUDY: PATIENT-DERIVED HEAD AND NECK CANCER XENOGRAFT ON MICE HUMANIZED WITH AUTOLOGOUS IMMUNE CELLS, A MODEL FOR PERSONALIZED IMMUNO-ONCOLOGY RESEARCH}

\footnotetext{
${ }^{1} \mathrm{M}$ Stecklum*, ${ }^{2} \mathrm{~K}$ Klinghammer, ${ }^{1} \mathrm{~A}$ Wulf-Goldenberg, ${ }^{1} \mathrm{~B}$ Brzezicha, ${ }^{3} \mathrm{~K}$ Jöhrens, 1J Hoffmann. 'EPO - Experimental Pharmacology and Oncology Berlin-Buch GmbH, Berlin, Germany; ${ }^{2}$ Charite University Medicine, Berlin, Germany; ${ }^{3}$ Universitätsklinikum Carl Gustav Carus an der Technischen Universität Dresden, Dresden, Germany
}

10.1136/jitc-2020-ITOC7.53 
Background The preclinical evaluation of novel immune modulators for cancer treatment remains a challenge, as models require both, engraftment of human tumor cells and a compatible human immune cells. In previous experiments, we have demonstrated, that we can use either peripheral blood mononuclear cells (PBMC) or hematopoietic stem cells (HSC) to establish a humanized immune system with functional T-, B-, and NK cells, monocytes, and dendritic cells. However these models are limited by rarely matching HLA isotypes between tumor and immune cells. In this case study, we established a patient-derived xenograft (PDX) model from a patient with Head and Neck squamous cell cancer (HNSCC). After engraftment of HNSCC PDX, patients PBMC were used to humanize mice. By this procedure we successfully generated a patient-specific human tumor-immune cell model in mice with 100\% HLA-match. Model development included the comparison of PDX engraftment on mice with either HLA-matching or non HLA-matching PBMC's and purified $\mathrm{T}$ cells from different donors. Furthermore, these effects were investigated on humanized mice generated with HSC. Finally, we further validated the model by comparing treatment effects with the checkpoint inhibitor Nivolumab in the autologous immune cell PDX model with heterologous models.

Methods The HNSCC PDX was transplanted on NOG mice. After tumor engraftment mice were randomized in 6 groups, receiving PBMCs by i.v. transplantation either from the patient or from 5 well characterized donors (PDX patient PBMCs 100\% HLA matching, 5 donors with different HLA matching). In the last step, PDX were transplanted on humanized mice generated from 5 different HSC donors. Blood and tumor samples were analysed by FACS and IHC for immune cell infiltration and activation.

Results In the autologous huPBMC model, no interference with the proliferation of HNSCC PDX was seen. However, on mice humanized with donor PBMC's with a high HLA match, a strong stimulation of tumor proliferation compared to non-humanized mice was observed. On humanized mice, generated from 5 different HSC donors, HLA-matching seem to have a lower influence on engraftment. On mice humanized with PBMC from different donors, we observed a correlation of treatment effects with HLA match, with strong tumor growth inhibition in the mice with the best match. In the PDX tumors, infiltrating immune cells were detected by FACS and IHC analyses.

Conclusions We developed a humanized immune-PDX model enabling appropriate preclinical translational research on tumor immune biology and the evaluation of new therapies and combinations, as well as the identification and validation of biomarkers for immune therapy. Furthermore, results showed a correlation between immune therapy effects and HLA matching in preclinical models.

Disclosure Information M. Stecklum: A. Employment (full or part-time); Significant; EPO - Experimental Pharmacology \& Oncology Berlin-Buch GmbH. K. Klinghammer: None. A. Wulf-Goldenberg: A. Employment (full or part-time); Significant; EPO - Experimental Pharmacology \& Oncology Berlin-Buch GmbH. B. Brzezicha: A. Employment (full or parttime); Significant; EPO - Experimental Pharmacology \& Oncology Berlin-Buch GmbH. K. Jöhrens: None. J. Hoffmann: A. Employment (full or part-time); Significant; EPO - Experimental Pharmacology \& Oncology Berlin-Buch $\mathrm{GmbH}$.

\section{P03.15 SITE-SPECIFIC IMMUNE EVASION AND SUBSTANTIAL HETEROGENEITY WITHIN ENTITIES PROVIDE EVIDENCE FOR PERSONALIZED IMMUNOTHERAPY}

${ }^{1} \mathrm{M}$ Thelen* ${ }^{*}{ }^{1} \mathrm{~K}$ Wennhold, ${ }^{1} \mathrm{~J}$ Lehmann, ${ }^{1} \mathrm{E}$ Staib, ${ }^{1} \mathrm{MA}$ Garcia Marquez, ${ }^{2} \mathrm{P}$ Lohneis, ${ }^{3} \mathrm{~A}$ Lechner, ${ }^{2} \mathrm{~S}$ Wagener-Ryczek, ${ }^{2,4} \mathrm{PS}$ Plum, ${ }^{5} \mathrm{D}$ Pfister, ${ }^{6} \mathrm{~F}$ Dörr, ${ }^{7} \mathrm{D}$ Beutner, ${ }^{8} \mathrm{~F}$ Thangarajah, ${ }^{8} \mathrm{D}$ Ratiu, ${ }^{8} \mathrm{~W}$ Malter, ${ }^{2} \mathrm{~S}$ Merkelbach-Bruse, ${ }^{9} \mathrm{CJ}$ Bruns, ${ }^{2} \mathrm{~A}$ Quaas, ${ }^{10} \mathrm{MS}$ von Bergwelt-Baildon, ${ }^{9,11} \mathrm{HA}$ Schlößer. ${ }^{1}$ Center for Molecular Medicine Cologne, University of Cologne, Faculty of Medicine and University Hospital Cologne, Cologne, Germany; ${ }^{2}$ Institute of Pathology, University of Cologne, Faculty of Medicine and University Hospital Cologne, Cologne, Germany; ${ }^{3}$ Department of Otorhinolaryngology, Head and Neck Surgery, Grosshadern Medical Center, Ludwig Maximilians University, Munich, Munich, Germany; ${ }^{4}$ Department of General, Visceral, Cancer and Transplantation Surgery, University of Cologne, Faculty of Medicine and University Hospital Cologne, Cologne, Germany; ${ }^{5}$ Department of Urology, University of Cologne, Faculty of Medicine and University Hospital Cologne, Cologne, Germany; ${ }^{6}$ Department of Cardiothoracic Surgery, University of Cologne, Faculty of Medicine and University Hospital Cologne, Cologne, Germany; ' Department of Head and Neck Surgery, University of Göttingen, Göttingen, Germany; ${ }^{8}$ Department of Gynecology, University of Cologne, Faculty of Medicine and University Hospital Cologne, Cologne, Germany; ${ }^{9}$ Department of General, Visceral, Cancer and Transplantation Surgery, University of Cologne, Faculty of Medicine and University Hospital Cologne, Cologne, Germany; ${ }^{10}$ Department of Internal Medicine III, University Hospital, LMU Munich, Munich, Germany; ${ }^{11}$ Center for Molecular Medicine Cologne, University of Cologne, Faculty of Medicine and University Hospital Cologne, Cologne, Germany

\subsection{6/jitc-2020-ITOC7.54}

Background Immune-checkpoint inhibition (CKI) demonstrated remarkable therapeutic efficacy in several kinds of cancer. However, immune escape mechanisms lead to primary or secondary resistance in the majority of patients. Most predictive biomarkers failed, as the primary target of CKI is not the tumor cell itself, but the crosstalk between immune- and cancer cells. We aimed to characterize the immune evasion landscape in primary tumors across different entities.

Materials and Methods Expression of 32 immune-regulatory molecules on lymphocytes was analyzed in peripheral blood and tumor infiltrating lymphocytes (TILs) of 146 primary tumor patients across 10 different entities using flow cytometry. NanoString was applied to determine RNA expression of the respective ligands and 20 genes associated with antigen presentation. Expression of coinhibitory ligands on tumor cells was assessed by immunohistochemistry. To quantify the immune cell infiltration, digital pathology was used and the Immunoscore was generated for each patient.

Results While an increase of regulatory $\mathrm{T}$ cells was a common feature across all entities, we found site-specific differences regarding other lymphocyte subsets and expression of immune-regulatory molecules by TILs and tumor cells. Expression of co-inhibitory molecules on tumor infiltrating $\mathrm{T}$ cells accumulated especially in advanced stage cancers whereas immune cell infiltration was mainly associated with enhanced antigen presentation. Co-expression of multiple immune-inhibitory ligands was most frequent in colorectal, lung and ovarian carcinoma. Genes related to antigen presentation were frequently dysregulated in seminoma, liver and lung cancer.

Conclusions Immune evasion is a common feature of cancer and frequently detected co-occurrence of multiple mechanisms probably contributes to resistance against immunotherapy. We describe substantial heterogeneity regarding immune escape mechanisms between patients with the same primary tumor. Individualized immunotherapeutic strategies based on pretherapeutic evaluation of the immune evasion landscape might help to improve response to CKI.

Disclosure Information M. Thelen: None. K. Wennhold: None. J. Lehmann: None. E. Staib: None. M.A. Garcia Marquez: 